\title{
Meta-Analysis of the association of IGF2BP2 gene rs4402960 polymorphisms with T2DM in Asia
}

\author{
Zhengchun Huang ${ }^{1}$, Minghua Dong ${ }^{2}$, Jian $\mathrm{Li}^{1}$, Wei Qiu${ }^{2}$ and Shumei $\mathrm{Li}^{2, a}$ \\ ${ }^{1}$ Department of Human Anatomy, Gannan Medical College, Ganzhou, Jiangxi, China \\ ${ }^{2}$ Department of Epidemiology in Preventive Medicine, Gannan Medical College, Ganzhou, Jiangxi, China
}

\begin{abstract}
In order to evaluate the association of IGF2BP2 (SNPS:rs4402960) gene polymorphism and type 2 diabetes mellitus (T2DM) susceptibility, we searched for all the related research literature by CNKI, Wanfan, Pubmed and Springer link database to collect data.Meta-analysis software(RevMan5.0) was applied for heterogeneity tests in genotype level, two methods(fixed-effects and random-effects model)were performed to pool the odds ratio(OR). Random-effects model was adopted if there were obvious heterogeneity among studies. On the contrary, a fixed effect model was used. At the same time, publication bias was examined by Funnel plot. The studies collected 28 articles(including 35 studies), and included 52277 cases of T2DM and 54168 controls. The pooled ORs of allele (T vs G)of rs 4402960 polymorphic loci in IGF2BP2 was significant association with T2DM (OR=1.163 95\% $\mathrm{CI}=[1.138,1.189] \mathrm{P}<0.00001)$.
\end{abstract}

\section{Introduction}

Type 2 diabetes mellitus (type 2 diabetes, T2DM), characterized by significant genetic heterogeneity, and are influenced by various environmental factors, resulted in peripheral tissue insulin resistance (IR) and insulin secretion deficiency, which lead to absolute or relative deficiency of insulin and high blood glucose by reducing uptake glucose [1]. With the new technologies like DNA sequencing, gene chip technique and other techniques application [2], they provide the new methods for researching T2DM susceptibility genes. Three different GWAS research groups in 2007 found that Insulin growth factor 2 mRNA binding protein 2 (insulin-like growth factor 2 mRNA binding protein 2, IGF2BP2) gene polymorphism and T2DM is associated[3-5]. Subsequently, multiple research groups carried out a lot of research about the association between T2DM and IGF2BP2, but research conclusions are not consistent in different populations from racial, ethnic, national, regional groups. Therefore, this research has comparative and cohort studies on associated cases published in Asia about relationships between the IGF2BP2 gene rs4402960 polymorphic loci and T2DM, with comprehensive evaluation the relationships between them, it offers valuable advice for high-risk T2DM screening and early prevention.

\footnotetext{
${ }^{a}$ Corresponding author: Shumei Li, gnyxylsm@163.com.

This study was supported by scientific fund from National Natural Science Fund in China (No.81360445).
} 


\section{Data and methods}

\subsection{Data sources}

The title Resources are from Joint search of CNKI, Wanfang database, and PubMed and Springer link database. The searching key words includes "T2DM, IGF2BP2, rs4402960 polymorphic loci, casecontrol study, chort study". The deadline for retrieval is May 31th, 2016.

\section{2 literature inclusion and exclusion criteria}

The literature included in the study was a case control study or cohort study; the study involved a number of areas of the world and the populations of races; Type 2 diabetes diagnosis were consistent with 1999 WHO diagnostic criteria and 2010 the American Diabetes Association (ADA) diagnostic criteria, patients were randomly selected regardless of age, sex, combined disease history, family disease history, and there were no special limit about years of research conducted or papers published. There are specific provisions on the content of sample; the original literature data provide OR value and $95 \% \mathrm{CI}$ or can be transformed into the OR value and $95 \% \mathrm{CI}$; literature contains enough information for comparison between T2DM patients and controls the IGF2BP2 gene rs4402960. Patients with type 1 diabetes, gestational diabetes, other special type of diabetes (insulin $\beta$ cell function of gene defects, insulin action gene defects, endocrine disease, etc.) are exclusive.

\subsection{Literature information extraction and statistical analysis}

\subsubsection{Data extraction and sorting}

The research related information are independently extracted from selected literature, and after data collection, they are filled in meta analysis of data abstraction, the information of the IGF2BP2 gene rs4402960 polymorphic loci and T2DM tables are formed, finally, the corresponding database are established according to the data from the original literature (OR and 95\% CI) .

\subsubsection{Heterogeneity test}

In order to explore whether there is a clear heterogeneity between the studies, the Q test based on the chi square test was used for quantitative analysis, that is, the use of $\mathrm{I} 2$ to measure the size of the heterogeneity [6]. If the study shows no statistical heterogeneity $(\mathrm{P}>0.1, \mathrm{I} 2<=25 \%)$, it will use the mantel Haenszel fixed effects model (Peto method) to merge data. If there is heterogeneity $(\mathrm{P}<0.1$, $25 \%<\mathrm{I} 2<50 \%$ ), the Dersimonian-Laird random effect model (D-L method) will be used to combine the data; if $\mathrm{I} 2$ greater than $50 \%$, it is thought that there is a obvious heterogeneity, subsequently, subgroup analyses exploring sources of heterogeneity is going to happen.

\subsubsection{The significance test of combined OR value}

Using the the $\mathrm{Z}$ value and $\mathrm{P}$ value in the part "test for the overall effect" in the RevMan5.20 software forest plots to do the significant test, if the calibration standard $P \leq 0.05$, we can consider it significant [7]. 


\subsubsection{Bias analysis}

Through the funnel plots to examine into the literature publication bias, if the funnel plot asymmetry may indicate the presence of publication bias, the funnel plot symmetry shows that there was no evidence of publication bias, the analysis is completed by statistical software RevMan5.20.

\section{Results}

\subsection{Basic information}

By searching, 28 articles conformed to associated cases control study about the IGF2BP2 gene rs4402960 polymorphic loci and T2DM are screened out, with 35 studies which contains 106445 people in seven Asian countries[6-33], including 52277 T2DM and 54168 controls .

\subsection{Meta analysis of the association between T2DM and IGF2BP2 gene rs4402960 polymorphism}

Figure 1 is the type of genes in the IGF2BP2 gene rs4402960 polymorphic loci (allele gene $\mathrm{T}$ vs $\mathrm{G}$ ) associated with T2DM and a meta analysis forest graph.

\begin{tabular}{|c|c|c|c|}
\hline \multicolumn{2}{|l|}{$\begin{array}{l}\text { Study } \\
\text { ID }\end{array}$} & \multirow{2}{*}{$\begin{array}{l}\text { ES }(95 \% \mathrm{Cl}) \\
1.37(1.17,1.60)\end{array}$} & \multirow{2}{*}{$\begin{array}{l}\% \\
\text { Welgh } \\
1.95\end{array}$} \\
\hline snintaro Omorl & - & & \\
\hline MIn Xu1,2, Yut & & $1.15(1.03,1.29)$ & 3.78 \\
\hline Ganes $h$ Chaunan & & $1.18(1.03,1.33)$ & 2.93 \\
\hline Ganes $n$ Chaunan & & $1.30(1.08,1.56)$ & 1.42 \\
\hline Maggle C.Y.Ng & & $1.05(0.93,1.18)$ & 3.38 \\
\hline Maggle C.Y.Ng & & $1.18(1.00,1.39)$ & 1.77 \\
\hline Maggle C.Y.Ng & & $1.24(1.09,1.42)$ & 2.74 \\
\hline Jle Wen1.. TIn & & $1.12(0.98,1.28)$ & 2.69 \\
\hline \multirow[t]{3}{*}{ Jonathan $\mathrm{T}$. Ta } & & $1.11(0.98,1.26)$ & 3.03 \\
\hline & & $1.00(0.89,1.12)$ & 3.63 \\
\hline & & $1.02(0.81,1.30)$ & 0.86 \\
\hline \multirow{4}{*}{$\begin{array}{l}\text { Yuklo Horkawa } \\
\text { Yasuharu Tabar } \\
\text { Xueyao Han? Y } \\
\text { Uma JyothI Kom }\end{array}$} & & $1.23(1.11,1.36)$ & 4.64 \\
\hline & & $1.24(1.01,1.52)$ & 1.16 \\
\hline & & $1.26(1.08,1.47)$ & 2.02 \\
\hline & & $1.10(0.95,1.28)$ & 2.16 \\
\hline Hur Hul Wu1, N & & $1.09(0.96,1.25)$ & 2.75 \\
\hline JlaHongxla,ect & & $1.12(0.89,1.40)$ & 0.93 \\
\hline Znang SM1. Xla & & $1.26(1.17,1.37)$ & 7.69 \\
\hline Rong Rong. R 00 & & $1.08(0.93,1.26)$ & 2.08 \\
\hline YIng Wu, 1 Huak & & 1.14 (0.95, 1.35) & 1.55 \\
\hline Xlao Ou snut". & & $1.28(1.12,1.49)$ & 2.35 \\
\hline M. Hork OShl \& & & $1.05(0.90,1.23)$ & 1.96 \\
\hline Fumink o Takeu & & $1.20(1.00,1.45)$ & 1.39 \\
\hline Fumink o Takeu & & $1.13(0.99,1.29)$ & 2.73 \\
\hline Fumink o Takeu & $\rightarrow$ & $1.14(1.07,1.21)$ & 12.67 \\
\hline Yong-Ho Lee & & $1.16(0.96,1.39)$ & 1.40 \\
\hline Y. LIU \& L. Yu & & $1.11(0.86,1.45)$ & 0.70 \\
\hline Fengliang Wel & & $1.35(1.13,1.61)$ & 1.55 \\
\hline Halnua Bal & & $1.21(0.97,1.50)$ & 1.01 \\
\hline Yun Qlan & & $1.15(1.00,1.32)$ & 2.49 \\
\hline Yl-cheng chang & - & $1.17(1.03,1.32)$ & 3.25 \\
\hline Neelam Hassana & & $1.10(0.97,1.25)$ & 2.98 \\
\hline Claudia H. T. & & $1.16(1.06,1.27)$ & 5.87 \\
\hline RaqueI Villega & $\rightarrow$ & $1.19(1.09,1.30)$ & 6.17 \\
\hline Qlong Huang & & $1.50(1.02,2.20)$ & 0.33 \\
\hline Overall (1-squared $-8.0 \%, p-0.334$ ) & $\diamond$ & $1.16(1.14,1.19)$ & 100.00 \\
\hline $\int_{.455}^{1}$ & & & \\
\hline
\end{tabular}

Figure 1. Meta analysis forest graph of rs4402960 IGF2BP2 gene polymorphism loci of genotype (T vs G) associated with T2DM. 


\subsection{Heterogeneity analysis}

Heterogeneity is an important link in the Meta analysis test, the purpose is to examine whether the results of independent research has can be merged. Rs4402960 gene in IGF2BP2 polymorphism loci genotypes ( $\mathrm{T}$ vs $\mathrm{G})$ has no obvious heterogeneity $(\mathrm{Q}=36.95, \mathrm{P}=0.334, \mathrm{I} 2=8.0 \%)$, it uses the fixed effect model to merger the OR values and 95\%CI. Results showed,the mergered OR values and $95 \%$ CI were $1.163(1.138,1.189)$. So it can be thought that rs4402960 gene in IGF2BP2 polymorphism loci of the allele $\mathrm{T}$ is the susceptibility genes of T2DM.

\subsection{The evaluation of publication bias}

From Figure 2, it can be seen that Meta analysis of inverted funnel figure of rs4402960 gene in IGF2BP2 polymorphism loci of the allele ( $T$ vs $G$ ) is basic symmetric, it is less likely to exist publication bias. And the Begg's Test and Egger's Test for Publication Bias also showed that both Pvalues were greater than 0.1 , it can be thought there is no publication bias.

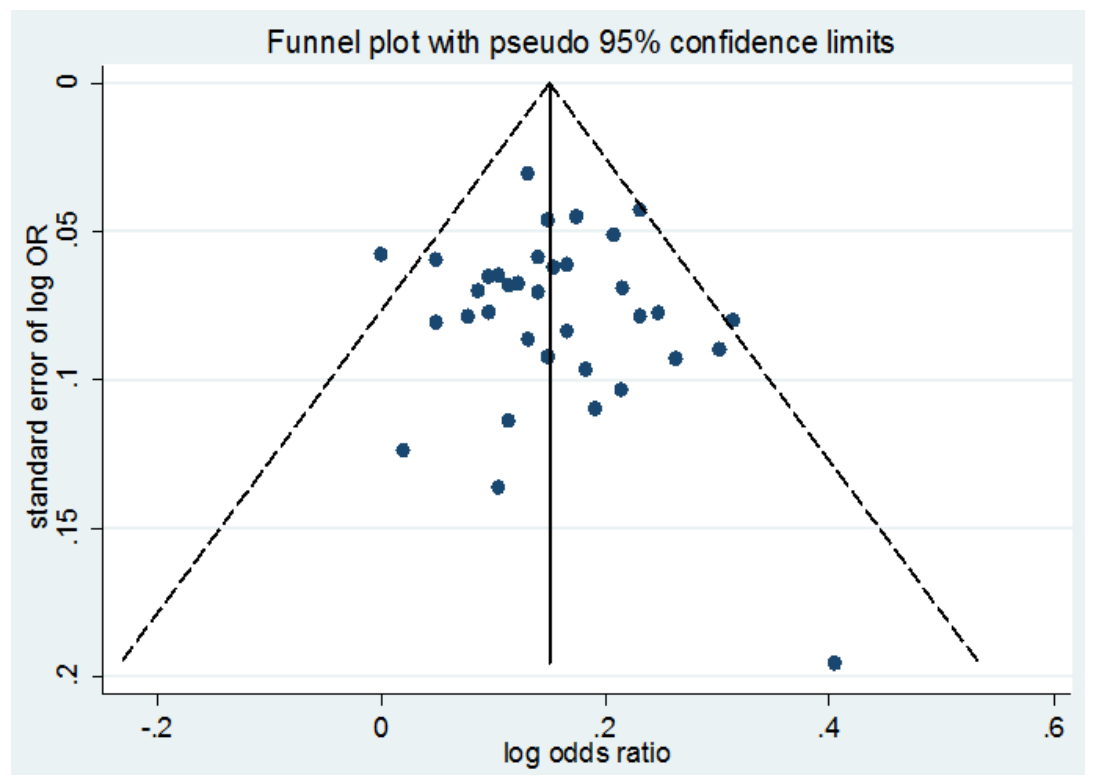

Figure 2. Bias evaluation funnel figure of rs 4402960 polymorphic loci alleles (T vs G) associated with T2DM.

\section{DISCUSSION}

From gene level and with the theory and methods of evidence-based medicine, this study evaluates the relevance between rs4402960 IGF2BP2 gene polymorphism loci and T2DM. The mergered OR value and $95 \% \mathrm{CI}$ of the allele ( $\mathrm{T}$ vs $\mathrm{G}$ ) were 1.163(1.138,1.189), it showed there are relevance between the rs4402960 IGF2BP2 gene polymorphism loci and T2DM. This study screening at home and abroad nearly all correlation research literature concerned with rs4402960 IGF2BP2 gene polymorphism loci and T2DM, and evaluate and deal with the quality of literature with scientific methods; then to these obvious heterogeneity between rs4402960 polymorphic loci alleles ( $T$ vs $G$ ). Results shows that there is no obvious heterogeneity in rs4402960 polymorphic loci alleles ( $\mathrm{T}$ vs $\mathrm{G}$ ) in Asia population. At the same time, bias may exist in every step of Meta analysis, so the study use the funnel figure to evaluate bias, it turns out that inverted funnel figures of IGF2BP2 rs4402960 polymorphic loci of genotypes and alleles are basic symmetric, indicating there is less likely to have a publication bias. 
The test results of this research may have bias to a extent, due to different detection technologies, different levels of detection and other inevitable errors within the study of the Meta analysis. The occurrence of T2DM has something with genetic, environmental and many other factors, this article only discusses the research of the correlation between rs4402960 IGF2BP2 gene polymorphism loci and the T2DM, however, other IGF2BP2 gene polymorphism loci are not involved, at the same time, such factors as diet, lifestyle, climate, environment may have interaction effect on T2DM, it is still necessary to further explore the molecular mechanism of IGF2BP2 result in T2DM.

\section{References}

1. Zai-ying Lu, Nan-shan Zhong editor, Internal medicine(People's Medical Publishing House, 2008 ).

2. Yan Chen, Guifeng Liu, Mei Wu.Chinese Journal of Gerontology 24,6346-6347(2013).

3. Saxena R, Voight BF, Lyssenko V, et al. Science 316,1331-1336(2007).

4. Scott LJ, Mohlke KL, Bonnycastle LL, et al. Science 316,1341-1345(2007).

5. Zeggini E, Weedon MN, Lindgren CM, et al. Science 316,1336-1341(2007).

6. Qiong Huang, Ji-ye Yin, Xing-ping Dai, et al.Acta Pharmacol Sin 31,709-717(2010).

7. Wu H-H, Liu N-J, Yang Z, et al. European Journal of Medical Research 19,40(2014).

8. Omori S, Tanaka Y, Takahashi A, et al. Diabetes 57,791-795(2008).

9. Xu M, Bi Y, Xu Y, et al. PLoS One 5( 2010).

10. Chauhan G, Spurgeon CJ, Tabassum R, et al. Diabetes 59,2068-2074(2010).

11. Ng MCY, Park KS, Oh B , et al. Diabetes 57,2226-2233(2008).

12. Wen J, Rönn T, Olsson A, et al. PLoS One 5(2010).

13. Tan JT, Ng DP, Nurbaya S, et al.J Clin Endocrinol Metab 95,390-397(2010).

14. Horikawa Y, Miyake K, Yasuda K, et al. J Clin Endocrinol Metab 93,3136-3141(2008).

15. Tabara Y, Osawa H, Kawamoto R, et al.Diabetes 58,493-498(2009) .

16. Han X, Luo Y, Ren Q, et al. BMC Medical Genetics 11,81(2010).

17. Kommoju UJ, Maruda J, Kadarkarai S, et al. Meta Gene 1,15-23(2013).

18. Hongxia Jia,Bin Gao, Yu Jie. The Chinese journal of clinical physicians 6,7528-7531(2012).

19. Zhang Si-min, Xiao Jian-zhong,et al. ChinMed J 126,4013-4018(2013).

20. Rong R, Hanson RL, Ortiz D, et al. Diabetes 58,478-488(2009).

21. Wu Y,Li H, Loos RJF, et al. Diabetes 57,2834-2842(2008).

22. Shu XO, Long J, Cai Q, et al. PLoS Genetics 6(2010).

23. Horikoshi M, Hara K, Ito C, et al. Diabetologia 50,2461-2466(2007).

24. Takeuchi F, Serizawa M, Yamamoto K, et al. Diabetes 58,1690-1699(2009).

25. Lee YH, Kang ES, Kim SH, et al. J Hum Genet 53,991-998(2008).

26. Liu Y, Yu L, Zhang D, et al. Diabetologia 51,2134-2137(2008).

27. Fengjiang Wei, Chunyou Cai, Shuzhi Feng,et al.Sci Rep 5(2015).

28. Haihua Bai, Haiping Liu, Suyalatu Suyalatu,et al.J Diabetes Res 6(2015).

29. Yun Qian, Feng Lu, Meihua Dong, et al.PLoS One 10(2015).

30. Yi-Cheng Chang, Pi-Hua Liu, Yu-Hsiang Yu, et al.PLoS One 9(2014).

31. Neelam Hassanali, N. Maneka G. De Silva, Neil Robertson, et al.PLoS One 9(2014).

32. Claudia H. T. Tam, Janice S. K. Ho, Ying Wang,et al.PLoS One 8(2013).

33. Raquel Villegas, Ryan Delahanty, Yu-Tang Gao,et al.PLoS One 7(2012). 